\title{
Design and measurement of a combined function magnet intended for a cancer therapy accelerator
}

\author{
Akio Morita, Yoshihisa Iwashita, Akira Noda, Toshiyuki Shirai, and Hiromu Tongu \\ Accelerator Laboratory, Nuclear Science Research Facility, Institute for Chemical Research, Kyoto University, Gokanosho, \\ Uji, Kyoto, 611, Japan \\ Masumi Umezawa and Kazuo Hiramoto \\ Power and Industrial Systems R\&D Laboratory, Hitachi Ltd., Omika, Hitachi, Ibaraki, Japan \\ Masahiro Tadokoro \\ Hitachi Works, Hitachi Ltd., Saiwai, Hitachi, Ibaraki, Japan \\ (Received 1 October 2001; published 12 December 2001)
}

\begin{abstract}
A compact proton synchrotron using combined function magnets is proposed to help realize the wider availability of charged particle cancer therapy facilities. This combined function magnet was designed with the help of three-dimensional magnetic field calculations to take account of a realistic fringe and the interference among the magnetic poles. An evaluation scheme for tune values based on particle tracking was developed to improve the magnet design. To verify the magnet design, a model magnet was fabricated and measured. In order to achieve a tune value evaluation from the measured magnetic field, schemes for accurate field mapping and field interpolation were developed. From the tune value evaluation of the measured magnetic field, it was thought that the performance of the model magnet was good enough to construct a synchrotron. In this paper, we report details of the design and the evaluation scheme for the combined function magnet and the results of the field measurements of the model magnet.
\end{abstract}

DOI: 10.1103/PhysRevSTAB.4.122401

PACS numbers: 41.85.Lc

\section{INTRODUCTION}

Radiation cancer therapy has recently been the subject of attention because of both its ability to preserve human body function and shape and its light load to the patient compared with other therapies. Among the radiation therapies, charged particle therapy has the advantage that it can localize dose distribution to the tumor, largely due to the presence of the Bragg peak which reduces damage to normal cells. But such charged particle cancer therapy is not yet common because of the high cost of the accelerator.

To realize widespread use of charged particle cancer therapy, an accelerator with low construction cost and easy handling is required. A compact proton synchrotron using combined function magnets is proposed as one approach for such an accelerator system [1,2].

Using combined function bending magnets as the main focusing elements, tracking between bending dipole and focusing quadrupole becomes unnecessary because the functions of bending and focusing are both realized with the same magnets. The operating point of the ring will be fixed if the relative distribution of the magnetic field does not change over the whole excitation range. As a merit of the combined function magnet, the possibility of eliminating a tune change due to a power supply ripple is pointed out and an experiment of proof of principle was achieved [3,4]. Power, coil space, and alignment costs between dipole and quadrupole can be saved. Using an untuned cavity $[5,6]$ and an rf source controlled by magnetic field strength, the acceleration operation will be easy.

However, the adjustment of the tune values after fabrication of such a magnet is not easy. To achieve a good magnet design we employed the three-dimensional magnetic field calculation code. To evaluate and adjust the tune values of the magnet, an evaluation method by particle tracking using calculated field distribution was developed.

In order to verify this design scheme, a model magnet was fabricated and its magnetic field distribution was measured. This paper describes our design of a combined function synchrotron emphasizing the design of the magnet and the measured results of the model magnet.

\section{DESIGN}

\section{A. Initial design}

The synchrotron ring is composed of six sets of $2 \mathrm{~m}$ drift space and $60^{\circ}$ sector magnets (Fig. 1). In the orbit in the bending magnet, the radius of curvature and the maximum field strength are designed as $1.9 \mathrm{~m}$ and $1.28 \mathrm{~T}$, respectively, for protons up to $240 \mathrm{MeV}$. The bending magnet has three combined function magnet sections and an FDF triplet focusing structure. The bending angles of the three poles are designed to be $15^{\circ}, 30^{\circ}$, and $15^{\circ}$. The design target values for horizontal and vertical tunes are both 1.75. After a preliminary calculation, the $n$ indices of the magnet poles $[n=-(\rho / B)(\partial B / \partial \rho)]$ were determined 


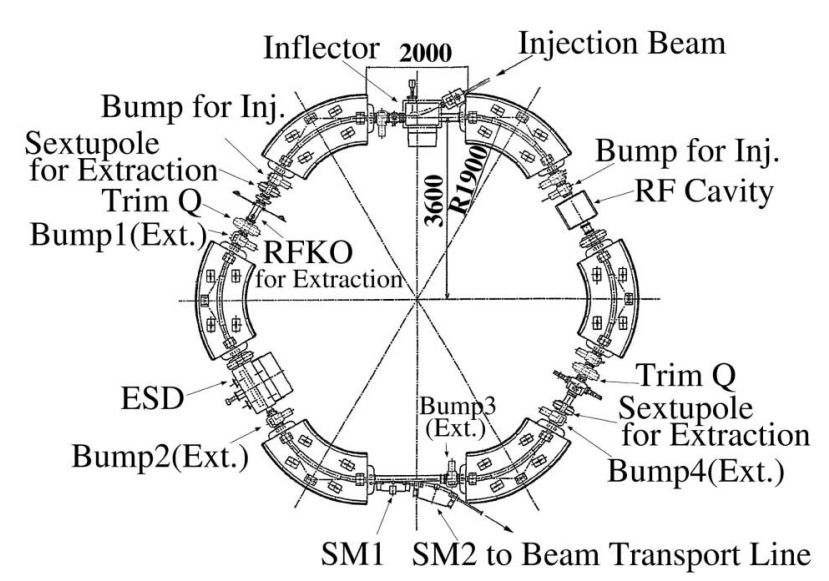

FIG. 1. Proposed compact proton synchrotron.

as -5.855 for the $\mathrm{F}$ sector and 6.164 for the $\mathrm{D}$ sector. The main coil is made by a hollow conductor, and the winding number of the main coil is 28 per pole. The conductors of the main coil make a $7 \times 4(\mathrm{~W} \times \mathrm{H})$ conductor stack, and this is installed into the magnetic pole with a one turn correction coil.

The basic magnet pole shapes are designed to suppress the sextupole component by a two-dimensional magnetic potential formula,

$$
\left[\frac{n}{2} \ln \left(1+\frac{x}{\rho}\right)+1+\frac{n}{2} \frac{x}{\rho}+\frac{n}{4}\left(\frac{x}{\rho}\right)^{2}\right] y=g,
$$
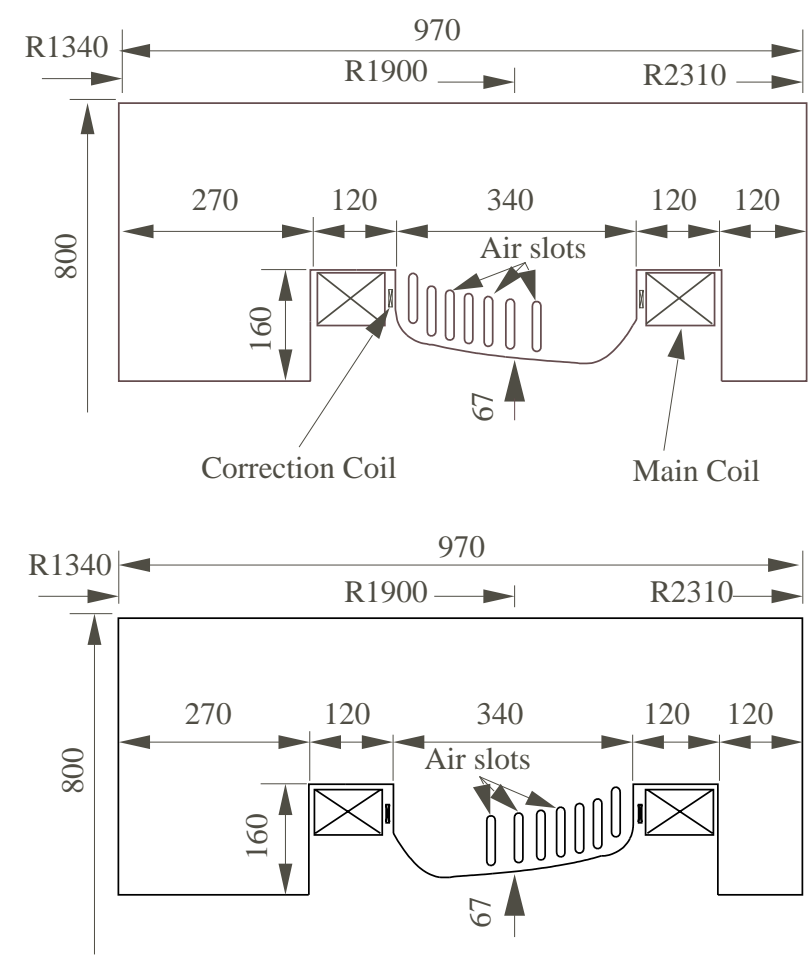

FIG. 2. Schematics of F and D sector poles.

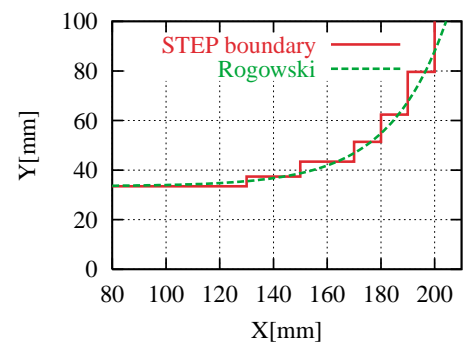

FIG. 3. (Color) Rogowski curve and simulating step function.

where $\rho, g$, and $n$ are the radius of the curvature of the magnet sector, the gap height on the designed orbit, and the $n$ index of the magnet pole, respectively. $x$ and $y$ denote the horizontal and vertical distance, respectively, from the designed orbit. The gap height on the designed orbit is $67 \mathrm{~mm}$. The sextupole error field is evaluated from a magnetic field distribution calculated by $2 \mathrm{D}$ code and its polynomial fitting. In order to avoid a variation of the field distribution due to saturation, slots to equalize the magnetic flux density [7] are situated at the low flux density area in the pole, as shown in Fig. 2. From the magnetic field calculations of the initial design, a useful aperture, $\pm 70 \mathrm{~mm}$, of the magnet with an error of $0.1 \%$ was obtained.

The entrances of the sectors are shaped by a step function which approximates the magnetic potential of the Rogowski cut pole. There are five steps. The width of these Rogowski-like steps are 10, 10, 10, 20, and $20 \mathrm{~mm}$, sequentially, from the edge of the sectors. Figure 3 shows the original Rogowski curve and the step function of the real pole cut.

\section{B. Operating point evaluation method}

To evaluate a realistic magnetic field including fringe fields and interference between the F and D sectors, the magnetic field distribution of the model magnet was calculated by the 3D magnetic field calculation code, TOSCA. A conventional analysis which used the transfer matrices of the ring component was not enough to evaluate the tune values from such a calculated magnetic field. Therefore we introduced a tune value evaluation method based on particle tracking [8]. In this method, the condition of the reference particle which made the closed orbit is determined from the given magnetic field at the first step. At the second step, a curvilinear coordinate $(s, x, y)$, which is based on the trajectory of the reference particle, is introduced and becomes the basis of the trajectory description. At the third step, a set of particles that has an initial distribution in a phase space is tracked in the magnet. Finally, the transfer matrices are reconstructed from the initial and final phase space distributions using least squares fitting.

From the curvilinear coordinate, the development of the trajectory is considered as the mapping of the phase space,

$$
m\left(s_{1} \mid s_{0}\right): \mathbf{x}_{0} \mapsto \mathbf{x}_{1},
$$


where $s_{0}$ and $s_{1}$ are the curvilinear coordinates of the initial and the mapped points, respectively, and $\mathbf{x}_{0}$ and $\mathbf{x}_{1}$ are the initial phase space vector at the point of $s_{0}$ and the mapped vector at the point of $s_{1}$, respectively. The linear component is the lowest order of this mapping because the origin is the fixed point of mapping from the definition of the reference orbit of the coordinate. In this mapping, the transfer matrix is understood as a linear representation. To extract the transfer matrix $M\left(s_{1} \mid s_{0}\right)$, we reconstructed it from the two phase space distributions by the following method. The initial vector of the particle number $n$ is $\mathbf{x}_{0}^{(n)}$ and the mapped vector of $n$ is $\mathbf{x}_{1}^{(n)}$; the least squares method for the transfer matrix is then written as

$$
\frac{\left.\partial \sum_{n}\left[\mathbf{x}_{1}^{(n)}-M\left(s_{1} \mid s_{0}\right) \mathbf{x}_{0}^{(n)}\right]\left[\mathbf{x}_{1}^{(n)}-M\left(s_{1} \mid s_{0}\right) \mathbf{x}_{0}^{(n)}\right)\right]^{T}}{\partial M}=0 \Rightarrow M\left(s_{1} \mid s_{0}\right)=\left[\sum_{n} \mathbf{x}_{1}^{(n)}\left(\mathbf{x}_{0}^{(n)}\right)^{T}\right]\left[\sum_{n} \mathbf{x}_{0}^{(n)}\left(\mathbf{x}_{0}^{(n)}\right)^{T}\right]^{-1},
$$

where $T$ denotes the transposition of the vector and the matrix. This reconstructed transfer matrix $M\left(s_{1} \mid s_{0}\right)$ is equal to the linear representation of the mapping $m\left(s_{1} \mid s_{0}\right)$ under the $L^{2}$ norm.

Once a transfer matrix of an arbitrary interval of the ring is calculated, we can obtain a transfer matrix of one revolution. The phase advance of betatron oscillation $\mu$ and the Twiss parameter $\alpha, \beta$, and $\gamma$ are easily obtained from the twodimensional transfer matrix of one revolution using the periodic boundary condition of the betatron oscillation in the following relationship:

$$
M_{\mathrm{rev}}(s)=\left(\begin{array}{cc}
\cos \mu_{\mathrm{rev}}-\alpha(s) \sin \mu_{\mathrm{rev}} & \beta(s) \sin \mu_{\mathrm{rev}} \\
\gamma(s) \sin \left(\mu_{\mathrm{rev}}\right) & \cos \mu_{\mathrm{rev}}+\alpha(s) \sin \mu_{\mathrm{rev}}
\end{array}\right)
$$

where $M_{\text {rev }}(s), \alpha(s), \beta(s)$, and $\gamma(s)$ are the transfer matrix of one revolution begun from the point $s$ and Twiss parameters at the point $s$, respectively. $\mu_{\text {rev }}$ denotes the phase advance of the betatron oscillation per revolution. Finally, the tune value $\nu$ is derived from the phase advance per revolution $\mu_{\text {rev }}$ as

$$
\nu=\frac{\mu_{\mathrm{rev}}}{2 \pi} .
$$

When we applied this method to our magnet, it was assumed that the closed reference orbit stayed on the median plane and coincided with the designed orbit at the middle of the drift space. With the help of these assumptions, we define the initial position and the initial direction of the reference orbit. The last parameter needed to complete the definition of the closed reference orbit is the initial momentum of the orbit. Therefore, a candidate of the closed reference orbit corresponding to the given initial momentum can be obtained by tracking the particle trajectory. Under good field symmetry, the closed reference orbit parallels the designed orbit at the center of the magnet. Thus, assuming such good field symmetry, the closed reference orbit is obtained by choosing the trajectory that parallels the designed orbit at the center of the magnet from the candidates generated by the tracking method. The angle between the designed orbit and the tracked trajectory at the center of the magnet is a function of the initial momentum of the trajectory. By applying the Newton-Raphson method to this function, the initial momentum generating the closed reference orbit is obtained. Once the initial momentum of the closed reference orbit is obtained, the curvilinear coordinate with respect to the reference orbit is defined and the calculations on the curvilinear coordinate can be performed. In order to make it easy to calculate the inverse matrices, the initial distribution of $\mathbf{x}_{0}^{(n)}$ was selected to eliminate the off diagonal terms of $\left[\sum_{n} \mathbf{x}_{0}^{(n)}\left(\mathbf{x}_{0}^{(n)}\right)^{T}\right]$ of Eq. (3). In the evaluation of the Twiss parameters and the phase advance, it was assumed that the betatron oscillations could be decoupled into $x-x^{\prime}$ and $y-y^{\prime}$ subphase spaces. This treatment, to decouple the betatron oscillations, is valid under the assumption of the magnet having good symmetric poles.

\section{Evaluation and tuning of the rough design}

The operating point in our rough design is evaluated as $\left(\nu_{h}, \nu_{v}\right) \sim(1.64,1.86)$. These tune values can be adjusted by modifying the ratio of bending angles between the $F$ and $\mathrm{D}$ sectors. This increases the bending angle of the $\mathrm{F}$ sector by $\Delta \theta$ degrees and decreases the bending angle of the $\mathrm{D}$ sector by $2 \Delta \theta$ degrees, as shown in Fig. 4. Because we plan to make the magnetic pole of the laminated iron sheets, this modification can be easily incorporated into the design by changing the number of the iron sheets laminated of the $\mathrm{F}$ and $\mathrm{D}$ sectors. Changing the ratio of the bending

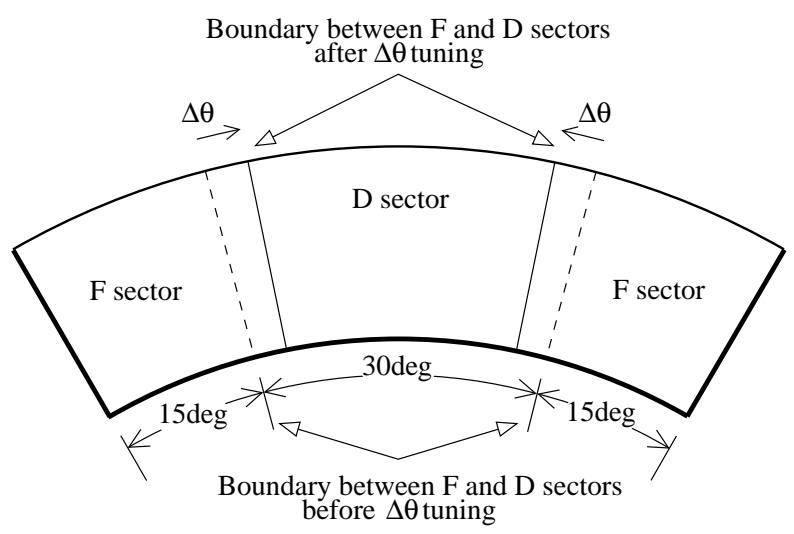

FIG. 4. Schematic of the bending angle modification in $\Delta \theta$ tuning. 


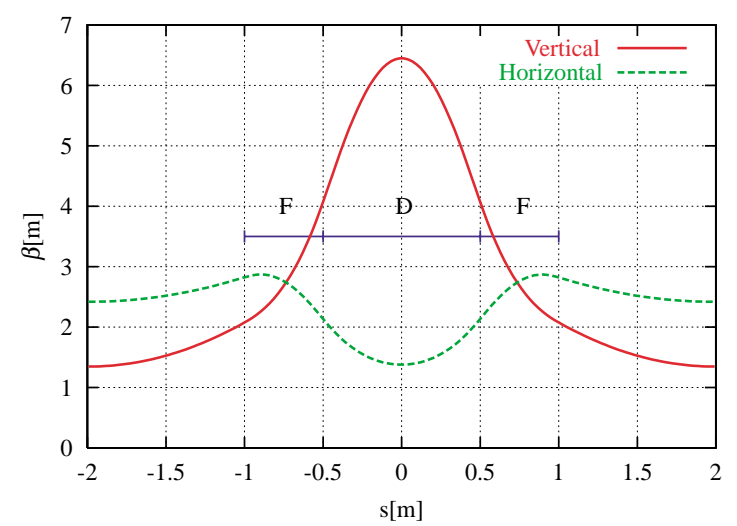

FIG. 5. (Color) Beta function of the lattice.

angles can be treated as a local modulation of the $n$ index around the transition region between the $\mathrm{F}$ and $\mathrm{D}$ sectors. From the tune shift formula of Hill's equation, the tune shift per bending angle modulation is obtained as follows:

$$
\begin{gathered}
\Delta \nu_{h} \sim-\frac{3}{\pi} \frac{n_{F}-n_{D}}{\rho} \beta_{h} \Delta \theta, \\
\Delta \nu_{v} \sim \frac{3}{\pi} \frac{n_{F}-n_{D}}{\rho} \beta_{v} \Delta \theta,
\end{gathered}
$$

where $n_{F}$ and $n_{D}$ are design $n$ indices and $\rho$ is the radius of the designed orbit curvature. $\beta_{h}$ and $\beta_{v}$ are horizontal and vertical beta functions at the border between the $\mathrm{F}$ and $\mathrm{D}$ sectors, respectively. Thus, under the linear approximation, the ratio of the horizontal and vertical tune shifts is given by the ratio of the beta function around the transition region, and the magnitude of the tune shifts is proportional to the modulation of the bending angle $\Delta \theta$. Figure 5 shows the beta functions evaluated from the calculated magnetic field distribution, where $\beta_{h}$ and $\beta_{v}$ in Eqs. (6) and (7) are about 2 and $4 \mathrm{~m}$, respectively. Thus the estimated proportional coefficients of the tune shifts for the angle $\Delta \theta$ are $0.21 \mathrm{deg}^{-1}$ horizontally and $-0.42 \mathrm{deg}^{-1}$

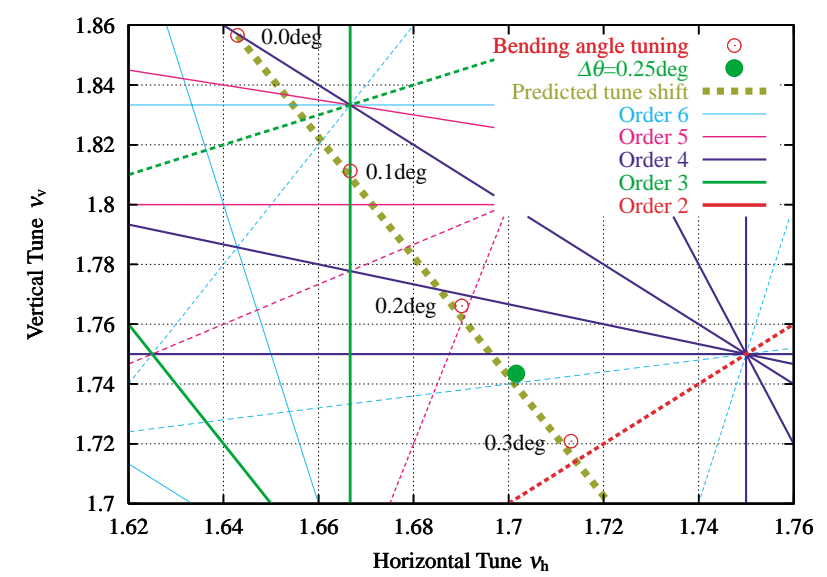

FIG. 6. (Color) $\Delta \theta$ dependence of tune values with resonance lines up to the sixth order. The solid lines and the dashed lines show the sum and differential resonance lines, respectively. vertically. The tune shifts predicted by Eqs. (6) and (7) are shown in Fig. 6 by the dotted line from the open circle labeled " 0.0 deg." The open and the filled circles in Fig. 6 show the evaluated tune values by the tracking method. These circles have good agreement with the predicted line. Therefore, using this $\Delta \theta$ tuning method, we can control the operating point on the predicted line, as shown in Fig. 6.

\section{Final design and model magnet}

Considering the operating point and resonance lines in Fig. 6 , we selected $0.25^{\circ}$ for the $\Delta \theta$ parameter and operating point $(1.70,1.74)$ of the final point to avoid resonance lines up to the fifth order. Figure 7 shows the excitation dependence of tune values evaluated from the magnetic field calculated by TOSCA with main coil current conditions of $383,574,765,861,957,1053,1148,1244$, and 1349 A. In Fig. 7, the horizontal tune is shifted to the lower side with an excitation increase, and the vertical tune has a peak around current $I=1000$ A. From the analysis of the position dependence of the contributions to the tune shift, the qualitative property of excitation dependence can be explained as follows. The ratio between the vertical and horizontal beta functions, which determine the modulation magnitude of the tune values, is especially large in the D sector. In the low excitation case, field deformation in the D sector is small, and the tune shifts are dominated by field deformation of the F sector. Then the vertical and horizontal tunes move in opposite directions. In the condition beyond the current of $1000 \mathrm{~A}$, a strong field deformation due to pole saturation appears in the $\mathrm{D}$ sector. This saturation affect dominates only the vertical tune, then the direction of the vertical tune shift is changed. Therefore it is easily supposed that the tune excursion curve is modified by the B-H curve of iron, the lamination packing factor, and the machining error of the slots equalizing flux density, etc.

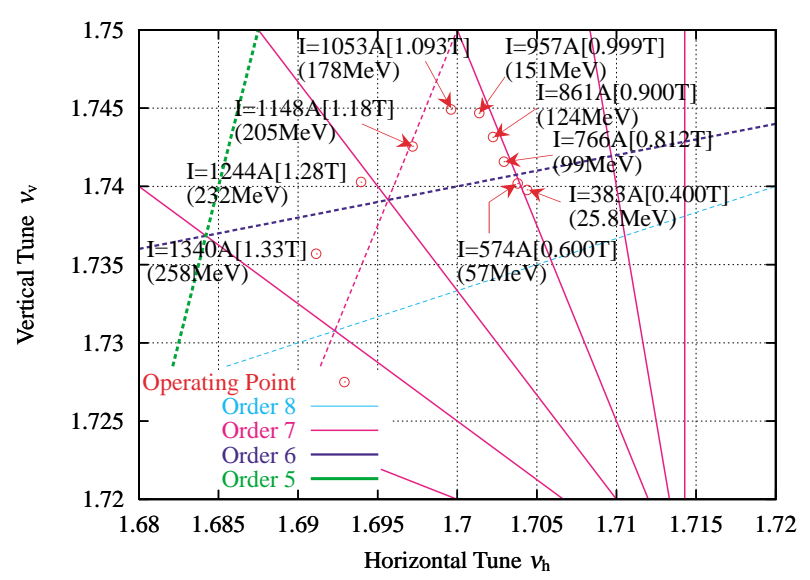

FIG. 7. (Color) Excitation dependence of tune values with resonance lines up to the eighth order. The solid lines and the dashed lines show sum and differential resonance lines, respectively. Low order resonance lines up to the fourth do not exist around the operating point. 


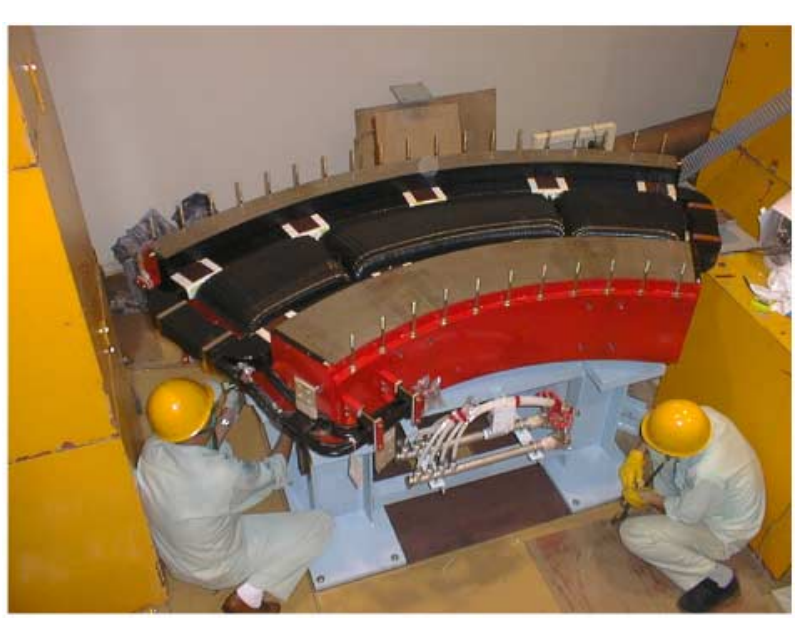

FIG. 8. (Color) Lower half of the model magnet.

To verify the three-dimensional calculation, we fabricated a model magnet reflecting the final design and measured the field distribution. Figure 8 shows the lower half of the model magnet being assembled. The poles of the magnet are made of laminated silica steel sheets of $0.5 \mathrm{~mm}$ thickness compatible with Nippon Steel Corporation 50H600 (see http://www.nsc.co.jp/si-steel/products/ 05.html). The achieved packing factor of the laminated pole was about 0.95 .

\section{FIELD MEASUREMENT}

\section{A. Basic strategy}

In order to check the $n$ index and the operating point of the model magnet, we needed accurate field gradient additions to the field strength. Because a harmonic coil method is not feasible for a sectored magnet, Hall probe mapping was chosen. This Hall probe mapping was achieved using the combination of a three-axes stage and Hall probes. The alignment method and the details of the measurement setup are described in a later section.

The information of field distribution required by the transfer matrix reconstruction using particle tracking is less than that in a full three-dimensional distribution, because of its linear approximation, magnet pole symmetry, and Maxwell law. Although evaluation of the dynamic aperture requires a full three-dimensional measurement, such a measurement is difficult because of the limitations of the measurable area, the probe stability, and the measurement time. Thus, we restricted measurement to the minimum set, which was enough to evaluate the $n$ index and tune values by tracking. The symmetry between the upper and lower poles and Maxwell equation $\nabla \cdot \mathbf{B}=0$ told us that the magnetic field flux crosses perpendicular to the median plane, and that the magnetic field near the median plane can be reconstructed from the major component $B_{z}$ on the median plane as follows:

$$
\begin{gathered}
B_{x}(x, y, z)=\left.\frac{\partial B_{z}(x, y, 0)}{\partial x}\right|_{z=0} z+O\left(z^{2}\right), \\
B_{y}(x, y, z)=\left.\frac{\partial B_{z}(x, y, 0)}{\partial y}\right|_{z=0} z+O\left(z^{2}\right), \\
B_{z}(x, y, z)=B_{z}(x, y, 0)+O\left(z^{2}\right) .
\end{gathered}
$$

\section{B. Alignment method}

Before field mapping, both the three-axes stage and the Hall sensor axis of the Hall probe have to be mechanically aligned against the magnet and the magnetic median plane found. To arrange the horizontal plane of the stage with the horizontal plane of the magnet, both the magnet and the stage are leveled using a water level. To arrange the movable area and the moving axes of the stage within the measurement region, the stage axis is aligned to the magnet axis at an appointed angle using a theodolite in the horizontal plane after translation of the stage. After mechanical stage alignment, the probe axes have to be aligned with the coordinate axes of the magnetic field to accurately measure the component of the magnetic field. It is difficult to find the coordinate axes of the magnetic field because of the change in the direction of the magnetic flux caused by the combined function magnet. Thus, a plane in which the direction of the flux is already known has to be found before alignment of the probe axes. Fortunately, the median plane has such a property because the whole magnetic flux is perpendicular to the median plane if the poles are symmetric. Because the flux direction in the offmedian plane depends on the $n$ index of the pole, the positional dependence of the flux direction over the whole measurement region can be detected by a roughly aligned probe. Thus the problem of finding the magnetic median plane is replaced with one of finding the plane where the directions of the flux of the F and $\mathrm{D}$ sectors are aligned. Using this searching method for the median plane, the accuracy requirement of the primary probe alignment can be relaxed. Once the median plane is found, the probe axes can be aligned with that plane and the alignment error of the probe size effect reduced by iterating the median plane searching and probe alignment. Finally, the probe origin in the horizontal plane is determined mechanically by using the geometry of the probe and the magnet.

\section{Setup and equipment}

It is difficult to measure a field map of a whole area with a single setup because of the sector shape of the magnet. Considering the mirror symmetry of the magnet along the beam path, we measured only half of the magnet area with a small overlap of the mirrored area. Figure 9 shows the geometry of the measurement setup. In this setup, the probe arm has a tilt angle of $15^{\circ}$ to the central orbit at the entrance, to cover the maximum magnet aperture of 


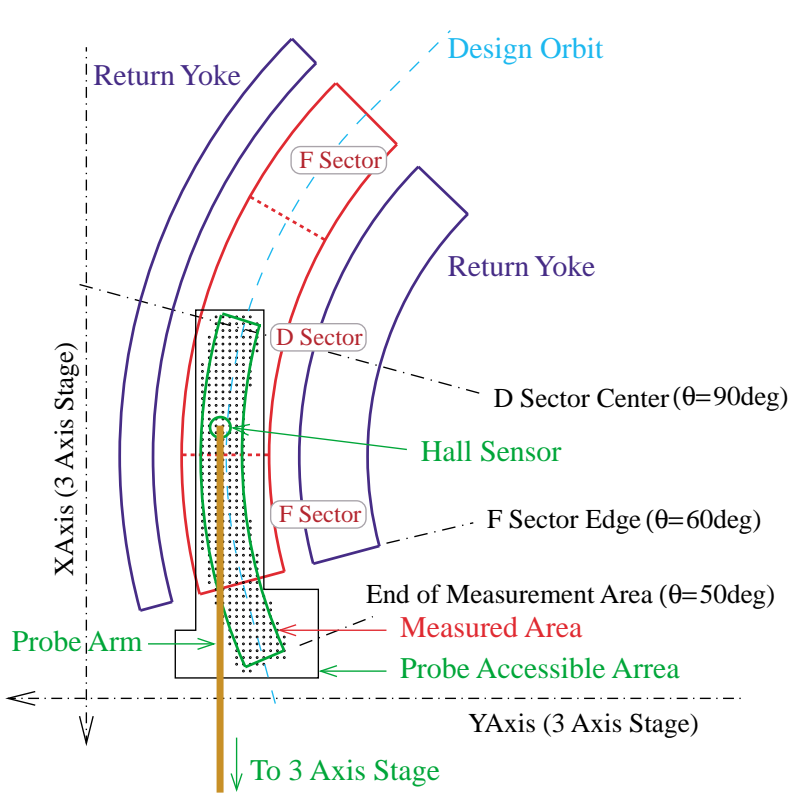

FIG. 9. (Color) Geometry of the measurement setup. The probe moves on a Cartesian grid in the "probe accessible area." The measurement is achieved in the "measured area."

the measurement area. The longest axis of the movable stage is aligned to the probe arm direction, which is used as the main scan axis to reduce the vibration of the probe arm when excited by the acceleration and deceleration of the stage. Figure 10 shows the setup as seen from the rear of the stage.

Measurement was performed on $5 \times 5 \mathrm{~mm}$ rectangular grids along the stage axes. Considering the requirements for the evaluation of the field characteristics, only the field distribution around the designed orbit is needed. The zonal area of the field distribution around the closed orbit, whose width is proportional to the beta function, is required to track the betatron particles. The beta function is maximized at the center of the $\mathrm{D}$ sector, and this position stays at the deepest area in the measurement region. Hence, the

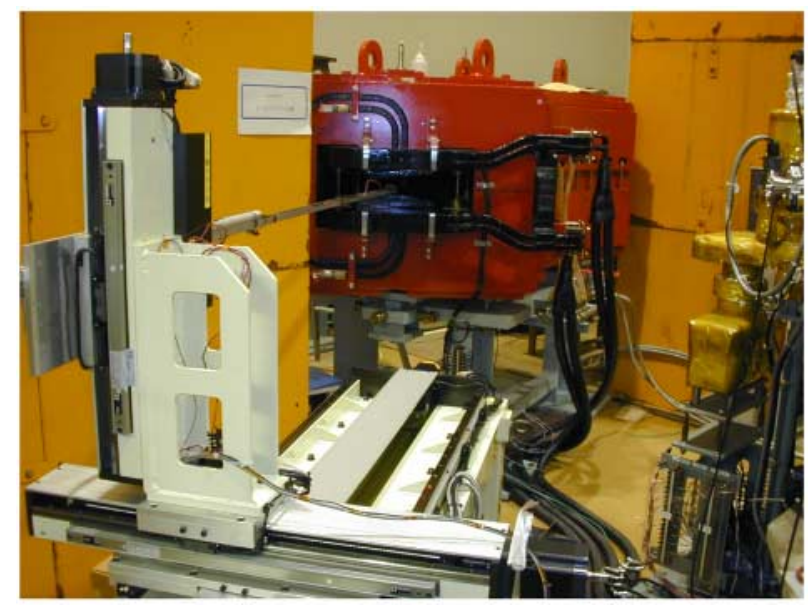

FIG. 10. (Color) Three-axes stage and model magnet aligned. scan width of this measurement area cannot be extended widely because of the conflict between the probe arm and the coil support. In order to reduce the measurement time, we decided to cut the measurement points which had relatively small contributions to the tracking aperture and the characteristic evaluation. Thus the selected measurement area covered half of the $\mathrm{D}$ sector, the $\mathrm{F}$ sector, and a $10^{\circ}$ fringe within a radius from 1.84 to $1.96 \mathrm{~m}$. The total number of the measurement points in the selected area was 7139.

Our method for searching the median plane requires a two- or three-axes Hall probe. Two commercially available three-axes Hall probes could not satisfy our requirements for the measurement stability, reproducibility, and differential linearity. We finally decided to use a single Hall sensor Group 3 MPT-141 that has a small sensitive area, high enough accuracy, and good stability (see http://ourworld.compuserve.com/homepages/ group3tech/DTM.htm). Its sensitive area is a rectangle of $1.0 \times 0.5 \mathrm{~mm}$. The maximum temperature coefficient of the corrected readout is guaranteed within $\pm 10 \mathrm{ppm} /{ }^{\circ} \mathrm{C}$ according to the catalog specification. Two Hall MPT-141 sensors were mounted on the aluminum mount (see Fig. 11), in which the geometric center of the sensing areas of the two Hall sensors stayed on the same horizontal plane at a distance of $7.5 \mathrm{~mm}$. This probe mount was attached to the tip of the probe arm. To control the Hall sensor angle against the flux, the directions of the two Hall sensors were perpendicular to the probe arm. The probe arm was fixed on the three-axes stage, which was driven with a resolution of $5 \mu \mathrm{m}$ by three stepping motors. The position reproducibility of the motor system, measured by a linear scale with $5 \mu \mathrm{m}$ accuracy, was held within $20 \mu \mathrm{m}$ on the axis having the worst reproducibility. The stepping motor drivers of the three-axis stage were connected to a TUJI DENSHI PM4C four-channel pulse motor controller (see http://www.tsuji-denshi.co.jp/english/ pm4c05a.html). All measurement devices were controlled

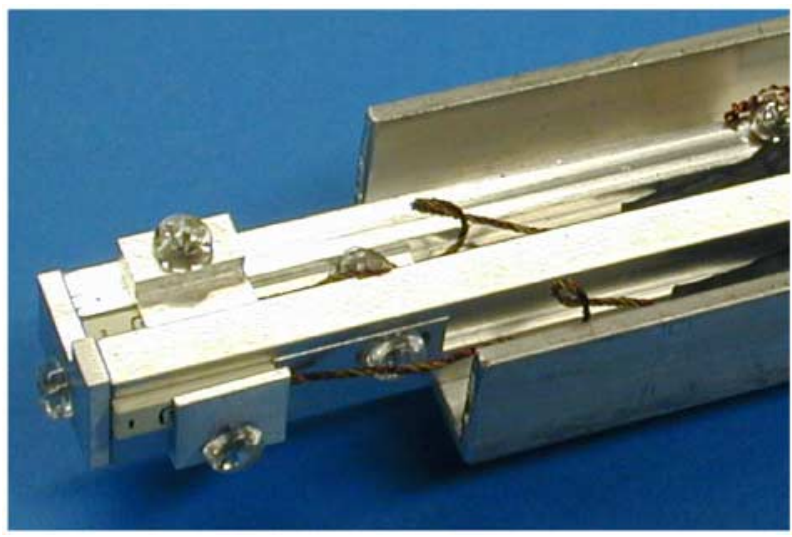

FIG. 11. (Color) Two Hall sensors MPT-141 installed on a twoaxis holder. The axes of the Hall sensors intersect at right angles to each other. 
by a Note PC via a NI PCMCIA-GPIB card (see http:// www.ni.com/catalog/pdf/1gpib756a.pdf). Even though the linear scalers installed in each axis were independent of the control system, it was useful to confirm that the motor system worked well.

\section{Field interpolation}

Evaluation of the field characteristics and particle tracking required a continuous smooth field distribution. The Fourier series expansion was chosen for interpolation because of its easy handling of derivatives. Using a Cartesian measurement grid, a Fourier series of a field distribution was obtained by two-dimensional discrete cosine transformation (2D DCT). Although all the measured points were on rectangular grid points, as previously described, not all grid points in the rectangular area had data because of the sector shape of the magnet. A grid point that had no measured data was treated as a free parameter with an initial value of zero. The following iterations were applied to reduce the number of Fourier components and noise. First, the Fourier components were obtained by DCT. At the second step, higher frequency Fourier components were reduced by a low pass filter (LPF). At the third step, the field map was reconstructed by inverse DCT (IDCT), including the missing data points. At the final step, the interpolation error was estimated on the measured grid points, and the calculated data in the measured area were replaced by measured values. The sequence DCT, LPF, IDCT, and refilling were iterated until the interpolation error came within tolerances.

\section{RESULTS OF MEASUREMENTS}

\section{A. Characteristics of the model magnet}

The excitation curve was initially compared with the TOSCA calculations. The excitation curve at the center of the D sector is shown in Fig. 12. The open and the closed

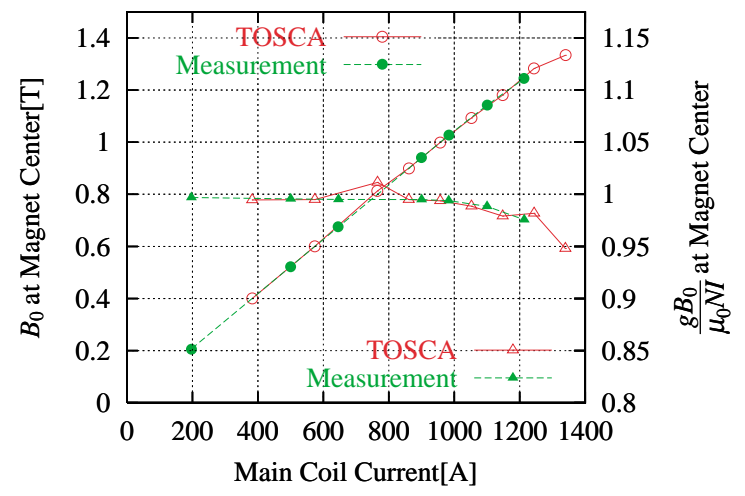

FIG. 12. (Color) Excitation curve of measured and calculated magnetic fields. The $B_{0}$ is a nominal value of the center of the D sector. The circles show the nominal value $B_{0}$. The triangles show the field strength per excitation power $g B_{0} / \mu_{0} N I$.

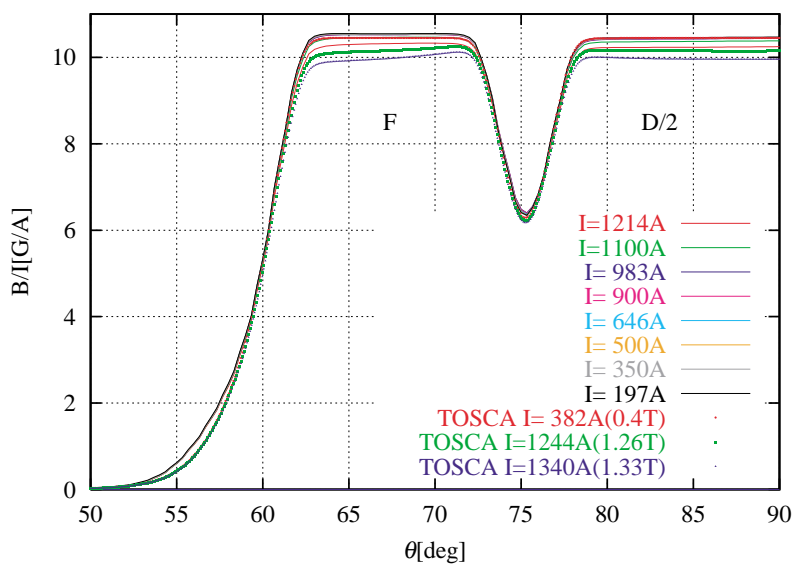

FIG. 13. (Color) Current normalized field strength distribution.

circles show the results of magnetic field calculations and the results of field measurements, respectively. The excitation curve of the measurements almost corresponds to the curve of the field calculations. The apparent threshold of the saturation phenomenon exists around the main coil current of $I=1200 \mathrm{~A}$. But the nonlinearity caused by magnetic pole saturation can be seen above a coil current of $I=900$ A from the curve of the field strength normalized by the coil current.
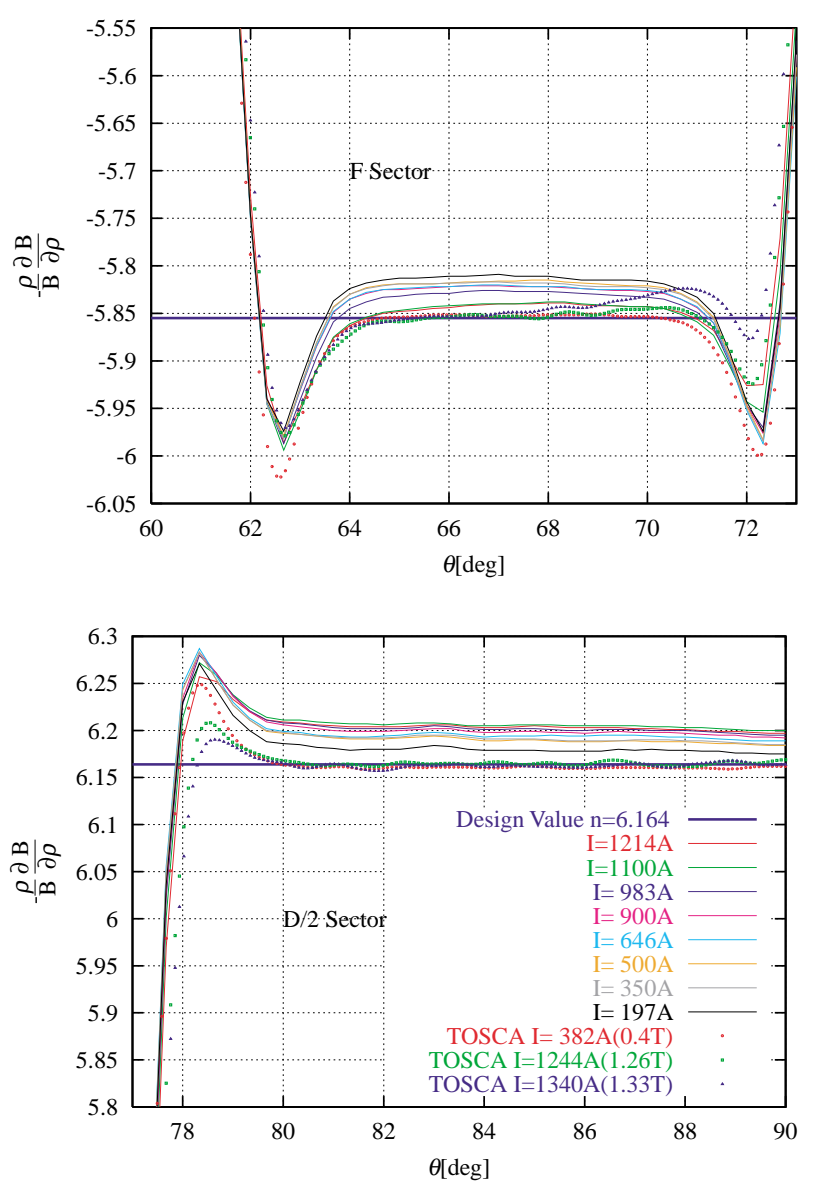

FIG. 14. (Color) $n$ index distribution. 
In order to compare characteristics of field distribution, the field distributions of TOSCA calculations were compared with the measured ones by evaluating the magnetic field strength normalized by the main coil current $(B / I)$ and the $n$ index $\left(-\frac{\rho}{B} \frac{\partial B}{\partial \rho}\right)$ distribution in the designed orbit.

Figures 13 and 14 show the normalized field strengths and $n$ indices, respectively, on an arc of $\rho=1.9 \mathrm{~m}$. This arc overlaps with the designed orbit of the magnet. Although the position of the magnetic field edge and the field strength for low field cases are well predicted by the calculation, the field strength of the fringe and the absolute value of the $n$ index are different.

The lamination structure of the pole edges was different between the model magnet and the TоSCA calculation model. In the TOSCA calculation, the whole magnet is constructed by sector lamination. But the Rogowski-like pole edge of the model magnet is laminated linearly because of assembling restrictions. This difference of the edge structure may be one of the causes of the characteristic difference around the magnet edges.

\section{B. Tune diagram of the measured result}

Previous graphs have shown the fidelity of the model magnet against the proposed design. The information is not enough to verify the operating point of the whole ring because of closed orbit distortion. Thus, we have to evaluate tune values by a tracking method based on the measured magnetic field distribution. Figure 15 shows an operating point evaluated by the tracking method. The field of the same excitation current was measured two times with a few days separation. The open circles show the primary measurement results and the filled circles show the secondary measurement results. These open and filled circles show the reproducibility of the measurements. The tune difference between the two measurements is larger in the lower field case because of relative error emphasis.

The major operating point was evaluated from the measurement as $\left(\nu_{h}, \nu_{v}\right) \sim(1.71,1.69)$. The horizontal and vertical differences of the major tune values between measurements and calculations are about 0.10 and -0.06 , respectively. This discrepancy can be explained as follows. The vertical tune has a large sensitivity to field gradient error because of the large beta function, as shown in Fig. 5.

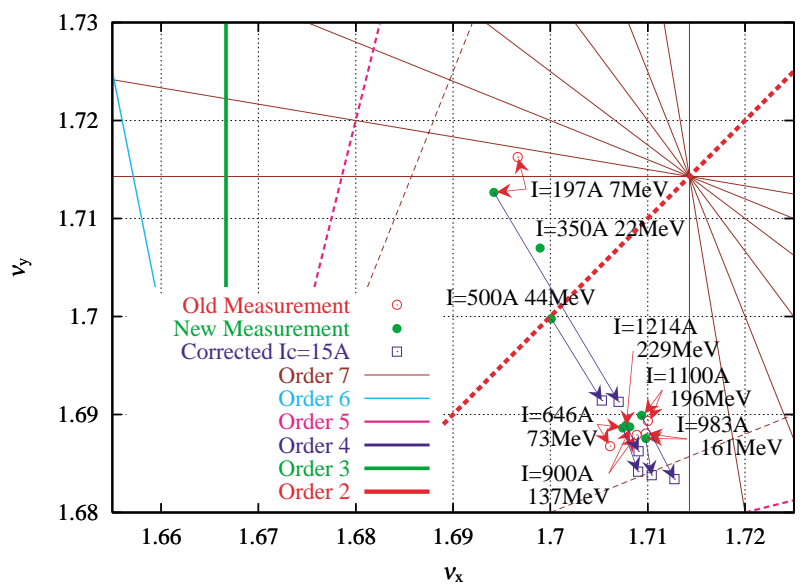

FIG. 15. (Color) Tune diagram from field measurement.

The focusing power of the fringe field, which is difficult to calculate accurately, affects only the vertical tune.

A large excitation tune shift is found in the low excitation case shown in Fig. 15, and the tune shift at a high excitation was relatively small. In the tracking in TOSCA's magnetic field, the tune shift is driven by the field deformation of iron saturation in the high magnetic field region. Thus, this measured tune shift does not agree with the suggestion of the tune value from the TOSCA evaluation shown in Fig. 7. Considering that the tune shift becomes large at lower excitation currents, it seemed that this tune shift was caused by an offset of the magnetic field. From simulations based on the measured field under this offset assumption, the expected offset of the magnetic field became about $5 \mathrm{G}$. But this value was too large to be explained by either zero offset of tesla meters or by the Earth's magnetism. Thus, it might be caused by the remanent magnetic field.

We tried to correct this tune shift with a one turn correction coil. This one turn correction coil was wound around the magnet poles and its winding directions were opposite between the $\mathrm{F}$ and $\mathrm{D}$ sectors, as shown in Fig. 16. Thus the correction coil could change the relative excitation level of the F sector against the D sector while keeping total bending power, because the total cross section of the correction current loop of the $\mathrm{F}$ sector was almost equal to the cross section of the correction current loop of the D sector. In a first order approximation, the closed orbit deformation was negligible and the tune shift was obtained from the tune shift formula of Hill's equation as

$$
\begin{aligned}
& \Delta \nu_{v} \sim-\frac{1}{4 \pi}\left(\int_{F} \beta_{v}(s) \frac{n(s)}{\rho^{2}(s)} d s-\int_{D} \beta_{v}(s) \frac{n(s)}{\rho^{2}(s)} d s\right) \frac{\Delta B}{B_{0}}, \\
& \Delta \nu_{h} \sim-\frac{1}{4 \pi}\left(\int_{F} \beta_{h}(s) \frac{n(s)}{\rho^{2}(s)} d s-\int_{D} \beta_{h}(s) \frac{n(s)}{\rho^{2}(s)} d s\right) \frac{\Delta B}{B_{0}},
\end{aligned}
$$

where $B_{0}$ and $\Delta B$ are the nominal value of the magnetic field in the designed orbit and the shift from the nominal value at the $\mathrm{F}$ sector, respectively. The integrals $\int_{F} d s$ and $\int_{D} d s$ are the integration on the whole of the $\mathrm{F}$ and $\mathrm{D}$ sectors, respectively. If the pole saturation is small, the ratio $\Delta B / B_{0}$ is proportional to the ratio of the ampere turn product between the correction coil and the main coil currents. 


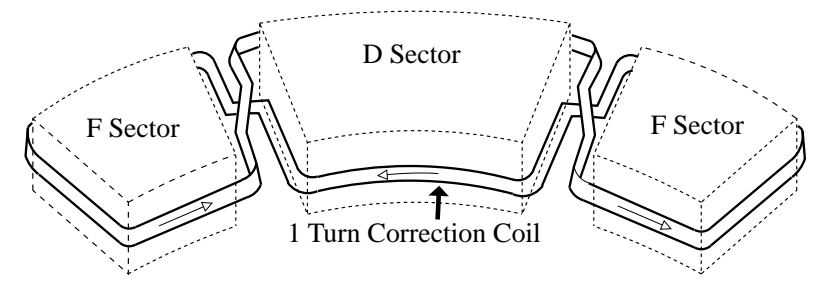

FIG. 16. Schematic of correction coil winding.

The open box in Fig. 15 shows the operating point of the magnetic field corrected by the correction coil with a constant excitation current of $15 \mathrm{~A}$. This correction is effective for the low excitation level; then the evaluated operating points come around the point $(1.71,1.69)$. The used correction current of $15 \mathrm{~A}$ is within the designed rating of the correction coil current of $100 \mathrm{~A}$. Thus, by using the correction coil, we could suppress the excursion of the operating point sufficiently to use for the synchrotron.

\section{SUMMARY}

We established an evaluation method for tune values from the magnetic field distribution and developed a field measurement scheme accurate enough to evaluate the tune values. The evaluated operating point from the measured field of the fabricated model magnet had reproducibility within 0.005 . The horizontal tune, which did not have a large error propagation from the magnetic field as did the vertical tune, agrees with the design value. In addition, tune fluctuation with current excitation was found, but it was confirmed that the tune fluctuations were easily compensated by a correction coil. Consequently, it was confirmed that we could fabricate a combined function magnet according to the design based on the three-dimensional magnetic field calculations, by paying attention to parameters which have large error propagations. We cannot yet confirm that a synchrotron ring works with this model magnet because a complete magnet set to make such a synchro- tron does not yet exist. However, from our tune value evaluation of the measured magnetic field, we think that the model magnet has sufficient performance for the construction of a synchrotron.

\section{ACKNOWLEDGMENTS}

The authors sincerely thank Dr. H. Ogawa, Dr. K. Noda, and Dr. M. Kumada at the NIRS for their support during field measurements. The authors also sincerely thank Dr. T. Kubo at KEK for his help in arranging a power supply unit for field measurements.

[1] A. Noda, Y. Fujita, K. Hiramoto, J. Hirota, M. Nishi, K. Noda, T. Shirai, M. Tadokoro, M. Torikoshi, E. Urakabe, S. Yamada, and M. Inoue, in Proceedings of the Sixth China-Japan Joint Symposium on Accelerators for Nuclear Science and Their Applications, Chengdu, China, 1996 (Institute of Nuclear Science and Technology, Sichuan, China, 1996), pp. 308-311.

[2] M. Tadokoro, J. Hirota, K. Hiramoto, M. Umezawa, and S. Kakiuchi, in Proceedings of the 1997 Particle Accelerator Conference, Vancouver, Canada (IEEE, Piscataway, NJ, 1997), pp. 3294-3296.

[3] K. Hiramoto and A. Noda, in Proceedings of the of the 11th Symposium on Accelerator Science and Technology, Harima Science Garden City, Hyogo, Japan, 1997 (SPring-8, Hyogo, Japan, 1997), pp. 496-498.

[4] A. Noda, A. Morita, K. Noda, M. Kumada, K. Hiramoto, and H. Kubo, in Proceedings of the 12th Symposium on Accelerator Science and Technology, Wako, Japan, 1999 (RIKEN, Wako, Japan, 1999), pp. 456-458.

[5] Y. Iwashita, Jpn. J. Appl. Phys. 36, L727 (1997).

[6] K. Saito, J. Hirota, F. Noda, Y. Iwashita, A. Noda, and M. Inoue, Beam Science and Technology 2, 15 (1997).

[7] Y. Iwashita, A. Noda, T. Shirai, A. Morita, M. Tadokoro, J. Hirota, M. Umezawa, and K. Hiramoto, Jpn. J. Appl. Phys. 38, L895 (1999).

[8] A. Morita, Y. Iwashita, and A. Noda, Beam Science and Technology 3, 23 (1998). 